FOR 00435

\title{
Neonatal respiratory distress secondary to nasal fibrous histiocytoma
}

\author{
Charles F. Koopmann ${ }^{1}$, Jr., Raymond B. Nagle ${ }^{2}$ and Robert Crone ${ }^{3}$ \\ ' Department of Otolaryngology - Head and Neck Surgery, The University of Michigan Hospitals, \\ Ann Arbor, MI 48109-0312 (U.S.A.); and Departments of ${ }^{2}$ Pathology and ${ }^{3}$ Otolaryngology, \\ University of Arizona Health Sciences Center Twcson, A2 85724 (U.S.A.) \\ (Received 10 December 1986) \\ (Revised version received 18 March 1987) \\ (Accepted 22 March 1987)
}

Key words: Neonatal respiratory distress; Nasal fibrous histiocytoma

\section{Summary}

A full term one-day-old neonate developed respiratory distress secondary to a right intranasal mass. After exploratory craniotomy revealed no intracranial lesions, the child was observed for 6 months. At that time he experienced severe apnea with cyanosis necessitating removal of the mass, which was diagnosed histologically as a fibrous histiocytoma. Seven year follow-up reveals no further problems. $\mathbb{A}$ discussion of fibrous histiocytoma of the nose and paranasal sinuses is briefly given.

\section{Introduction}

Respiratory distress in the neonate requires a systematic approach in the evaluation of the patient. Nasal obstruction secondary to a neoplasm or mass must be considercd as well as choanal atresia, laryngeal, subglottic, and tracheal and broncho-pulmonary causes. This case represents an intranasal fibrous histiocytoma leading to neonatal respiratory distress. The pathology and differential diagnosis as well as management is discussed.

Correspondence: C.F. Koopmann, Ir., Department of Otolaryngology-Head and Neck Surgery, Box 0312, Taubman Medical Center, 1500 East Medical Center Drive, Ann Arbor, MI 48109-0312, U.S.A. 


\section{Case report}

A six-month-old white male presented for evaluation and treatment of a right nasal mass first noted at the age of one day. At birth the child had excessive mucous from the right nares. One day later a swollen area along the right nasal region was noticed as well as noisy breathing. A head CT scan revealed a right intranasal mass

which was felt to be an encephalocele or hemangioma (Fig. 1). A neurosurgical consultant felt that the mass was most consistent with an encephalocele and recommended a craniotomy to look for an intracranial component as well as to see if there were any dural defects. At age 6 days, he underwent a craniotomy. No intracranial mass was found but an intranasal biopsy of the mass was interpreted as a benign hemangioma.

The baby was discharged from the hospital at age 21 days and observed. For 5 months he was maintained in an infant seat to protect against aspiration and apnea. At times he did require an oral airway because of apnea secondary to obstruction from his tongue. Due to progressive airway obstruction, increasing periods of apnea, and cyanosis, he was admitted to The University of Arizona Health Sciences Center for surgical removal of the lesion. Carotid angiography did not substantiate the

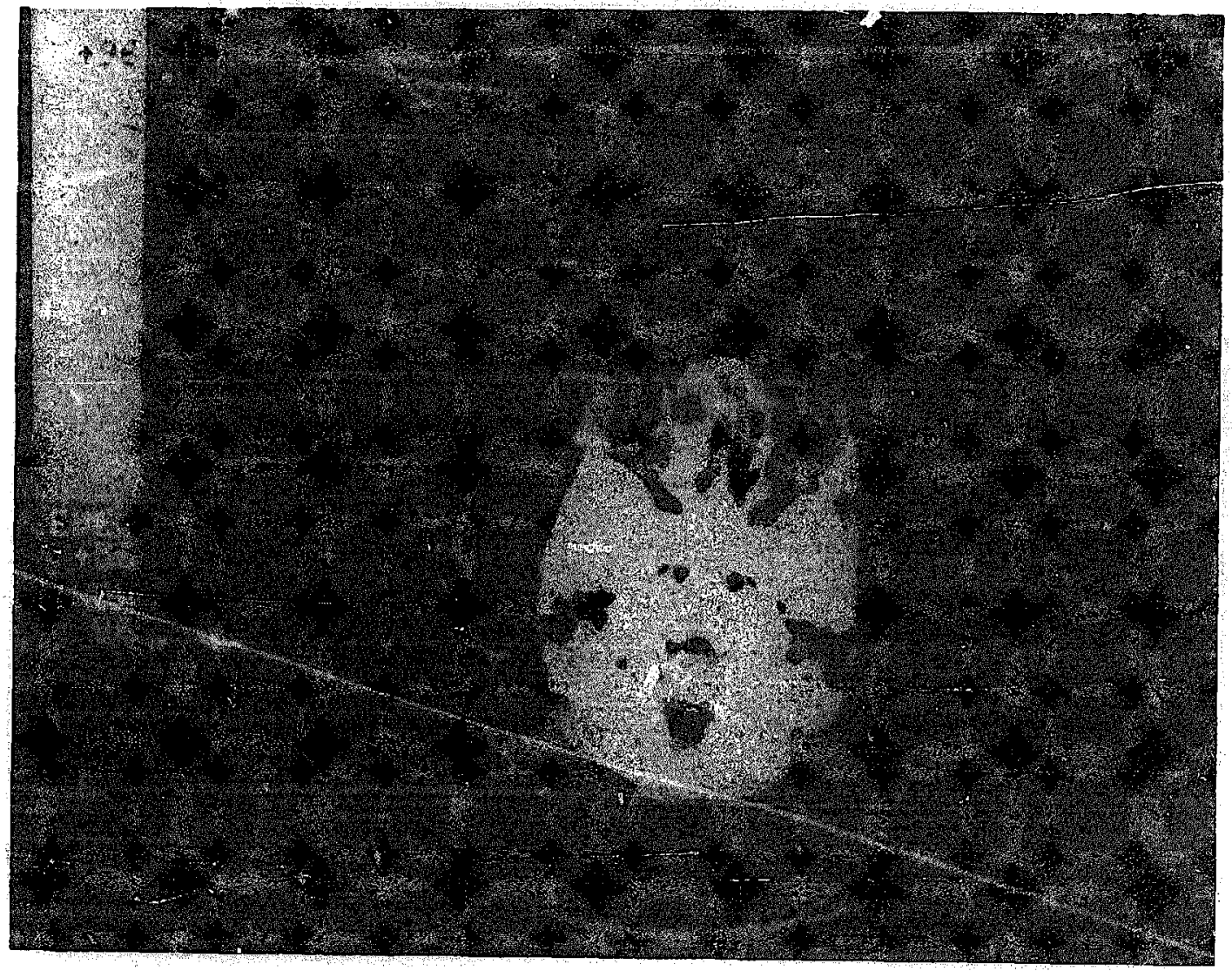

Fig. 1. CT scan which was interpreted as containing a right intranasal mass consistent with an encephalocele or hemangioma. 


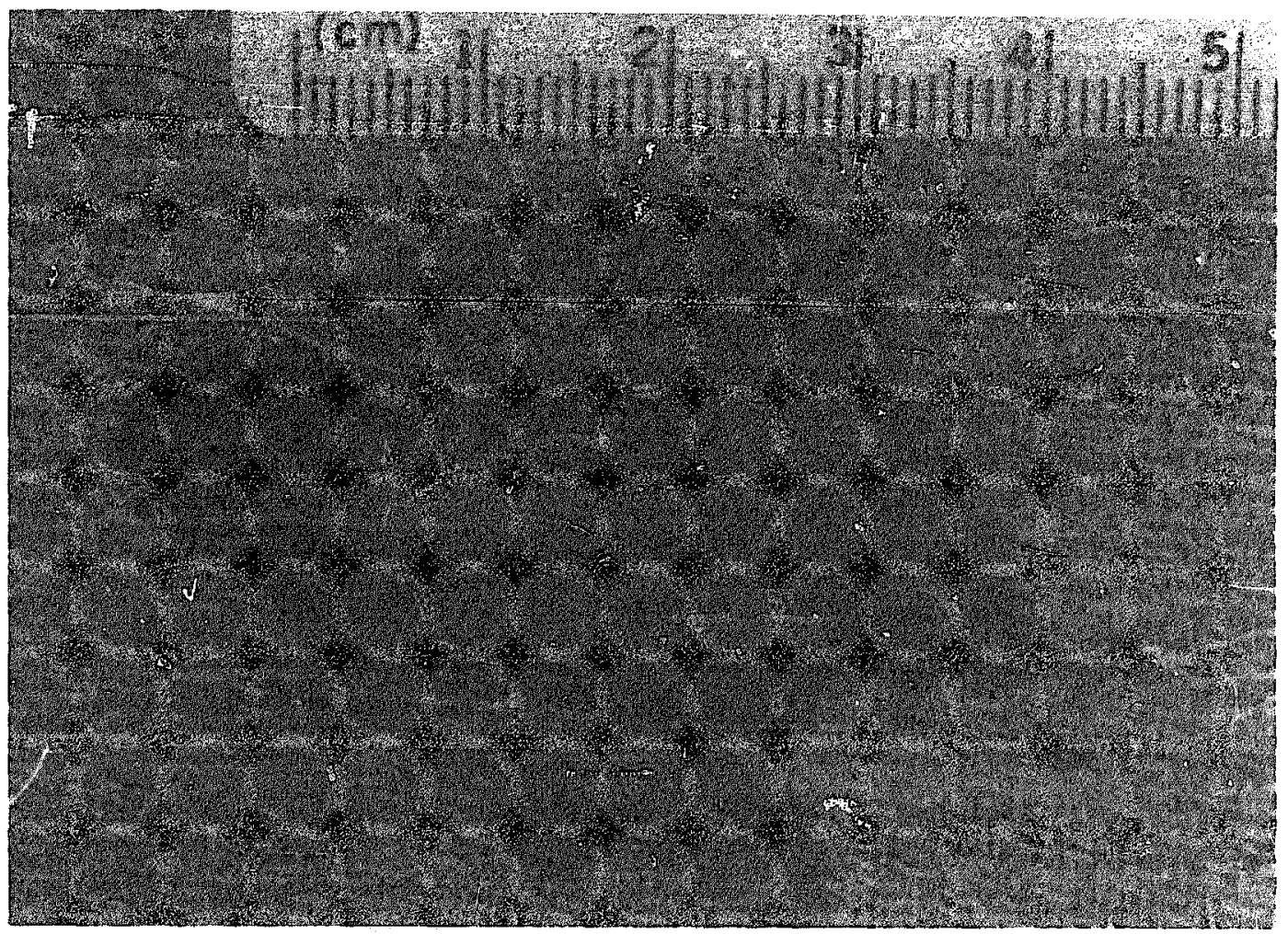

Fig. 2. The right intranasal mass after complete surgical removal.
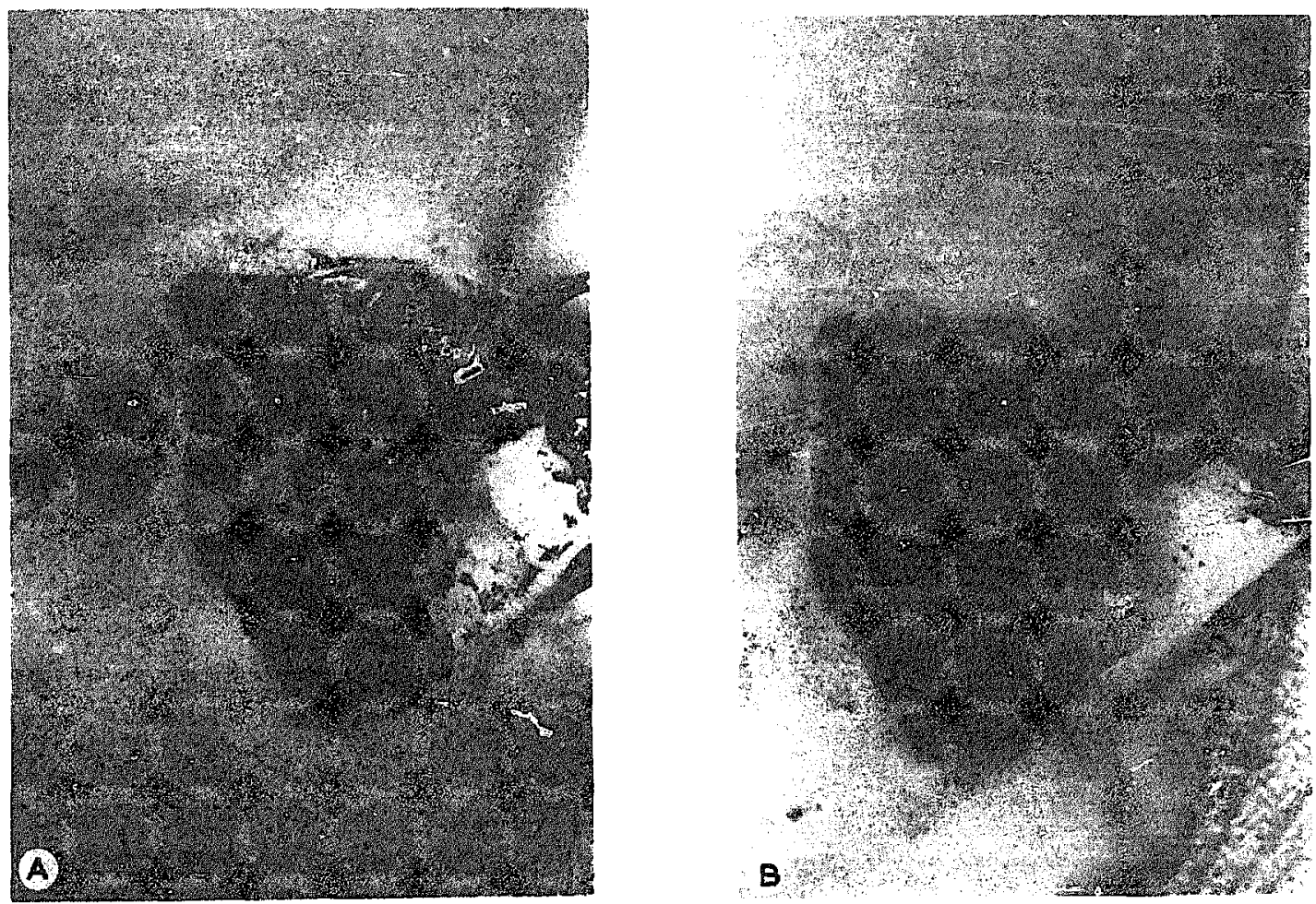

Fig. 3. A: intra-operative photograph illustraing the right obstructing intranasal mass. B: intra-operative photograph after removal of the intranasal fibrous histiocytoma illustrating the displacement of the nasal septum to the patient's left side. 

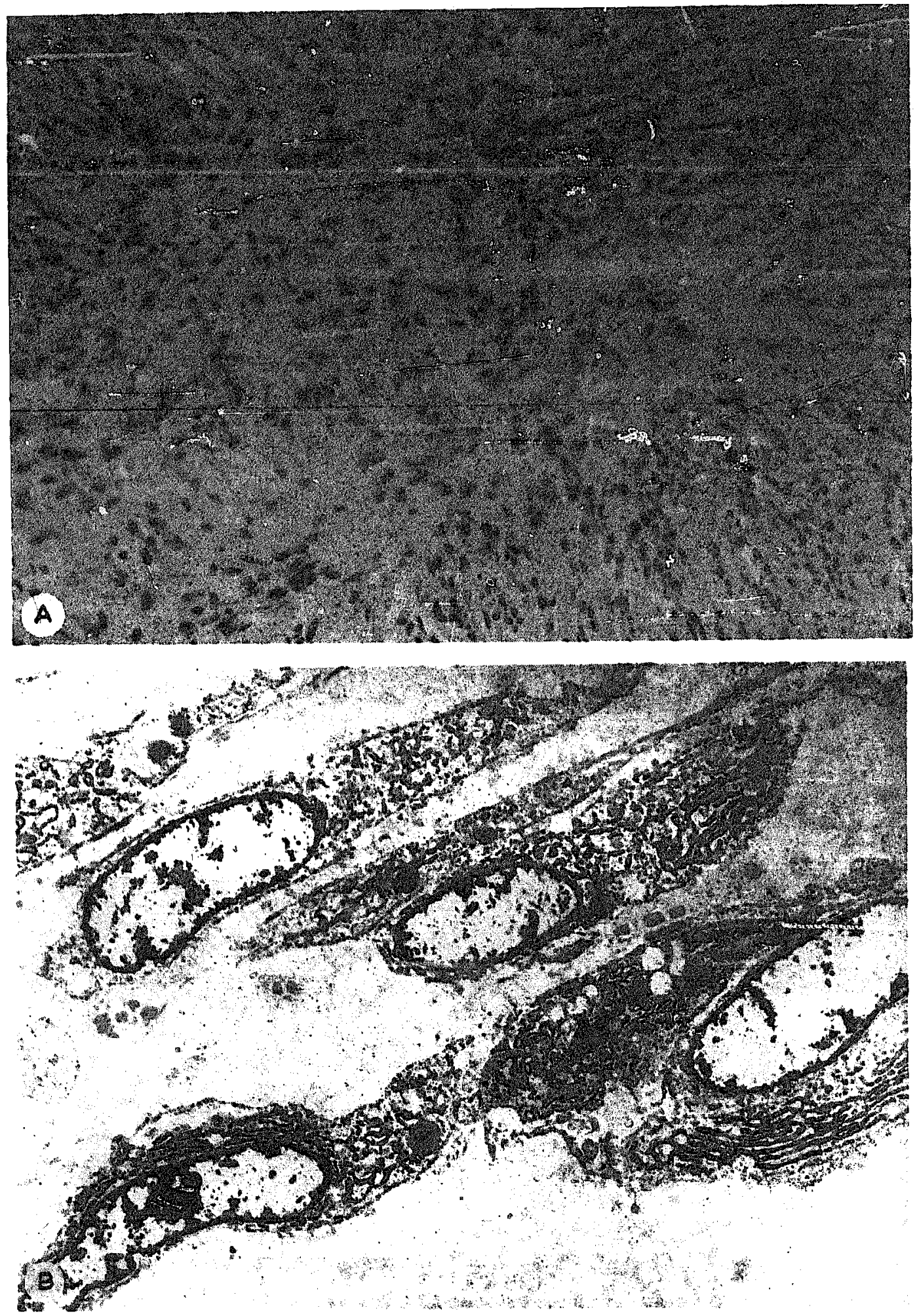

Fig. 4. A: routine histological sections revealing spindle shaped cells with elongated nuclei and infrequent mitotic figures. The fitrous tissue component has a dense collagen matrix. B: electron micrographs show the cellular nature of the neoplasm. The cells are spindle shaped cells with abundant cytoplasm containing numerous profiles of rough surfaces endoplasmic reticulum. The cells also contain scattered lipid vacuoles. There is no evidence of primitive sarcomere formation. Magnification: $A, \times 4725 ; \mathrm{B}$, $\times 4410$. 
diagnosis of a vascular neoplasm. Thus, the child underwent an excision of the mass via a lateral rhinotomy approach. The lesion (measuring $4 \times 2 \times 1.5 \mathrm{~cm}$, Fig. 2) was attached to the inferior turbinate and extended into the lamina papyracea of the ethmoid complex laterally, to the cribiform plate superiorly, and displaced the nasal septum to the left (Fig. 3). Total surgical removal was ascomplished with no intraoperative or post-operative complications. The child has been discase-free for 7 years. The final pathologic diagnosis was benign fibrous histiccytoma (Fig. 4).

\section{Discunssion}

Fibrous histiocytomas of the nose and paranasal sinuses are relatively unusual in all age groups and are extremely rare in neonates. In 1961 Kauffmann and Stout [4] reported on 15 children with histiocytoma with 9 arising between the ages of 0 and 5 years. None were in th: nose, paranasal sinuses, or nasopharyax. In 1973, Townsend et al. [7] reported the first known case of a fibrous histiocytorsa of the paranasal sinuses in a 3-year-old boy. The mass had been present for one year and had caused blunting of the left nasolabial fold as well as displacement of the nasal septum. $\mathbb{R i c e}$ et al. [5] reported two cases of fibrous histiocytomas of the nose and paranasal sinuses, one case being in a 13-year-old girl.

In 1980, Del-Ray and De-La-Torre [3] reviewed the world literature and found 7 fibrous histiocytomas reported in the nasal fossa and paranasal sinuses. In their case report, the initial pathological diagnosis was angiosarcoma although malignant angiofibroma, hemangiopericytoma, and fibrosarcoma were also considered. However, detailed ultrastructural and histochemical analysis led to the diagnosis of fibrous histiosytoma due to a storiform pattern of the fibroblasts.

In 1973, Shearer et al. [6] reported neonatal respiratory distress caused by a nasopharyngeal fibrous histiocytoma. This patient presented with respiratory distress at age one day when a nasopiaryngeal mass was discovered. As in our case a diagnosis of an encephalocele was made but a craniotomy revealed no anterior fossa mass. It should be emphasized that some neurosurgical consultants prefer to proceed with a craniotomy to look for intracranial involvement or dural iefects before any intranasal surgery is performed. Indeed, these neurosurgeons feel that if the intracranial portion is removed and the dura repaired, the intranasal portion will disappear without any rhinological removal. They fear intranasal biopsy and/or removal prior to or concurrent with intracranial procedures will increase the risks of meningitis and its sequelae. Definitive removal was postponed until age 8 months, when a lateral rhinotomy was utilized to remove a $3.5 \times 2.0 \mathrm{~cm}$ mass attached to the middle turbinate and to the cribiform plate. No follow-up was given.

According to Blitzer et al. [2], fibrous histiocytomas of the head and neck most commonly occur in the following locations (in decreasing order of frequency): (1) paranasal sinuses (maxillary, ethmoid); (2) mandible; (3) larynx; (4) neck. In this review of 87 cases, two were in the nose, one in the nasopharynx, and 5 in the ethmoid sinuses. They found that the prognosis improves with younger patients or 
in females. These authors recommend wide local excision, finding that tumor size, rather than rate of tumor growth, was an important prognostic factor.

Fibrous histiocytomas are soft tissue neoplasms composed of histiocytes and fibrous tissue. Histologically the fibroblasts will be aligned in either a storiform or nebula pattern and if spindle cells predominate, the diagnosis of fibrosarcoma may be made. The histiocytes may be seen as either multinucleated giant cells or found with a foamy cytoplasm. When histiocytes predominate, a granulomatous or inflammatory process may be diagnosed. Election microscopy will show that the neoplasm consists of cells which are spindie shaped and rather sparsely distributed. The cytoplasm shows abundant rough surface endoplasmic reticulum but there is no evidence of primitive sarcomere or other diagnostic ultrastructural features. Myelin figures are present in some cells and lipid vacuoles are seen. There are occasional intermediate type junctions seen between adjacent cells but there is no evidence of epithelial development. The intervening stroma contains fine bundles of collagen i toluding some long spacing collagen. There is no evidence of basement membrane forming around individual cells (Fig. 4).

These tumors may be extremely confusing and may be diagnosed as reticulohistiocytonias, histiocytomas, xanthomas, xanthogranulomas, fibrous xanthomas, sclerotic hemangiomas, progressive recurrent dermatofibromas, or dermatosarcoma protruberans among other diagnoses. Although approximately $1 \%$ of fibrous histiocytomas are malignant [6], a prediction of the biological behavior is difficult. Tumors with quite similar histological patterns may vary in behavior from being cured by simple resection to either recurring with local, aggressive growth or by metastasizing distally. According to Batsakis [1], such variable behavior is due to the histiocytes, with little correlation found between the number of mitoses and the degree of aggressiveness. Thus, an accurate prognosis is difficult to make.

\section{Conclusion}

The authors would like to emphasize that neonates in respiratory distress be carefully evaluated for intranasal neoplasms as well as for choanal atresia, laryngeal, subglottic, pulmonary, or tracheo-bronchial anomalies. Secondly, although they are essential, CT scans may be misleading as to intracranial extension. The authors would hope that such case reports as this (and Shearer et al. [6]), could be utilized to modify the approach of early craniotomy without definitive proof of intracranial extension. Thirdly, although delayed, definitive surgical intervention has been recommended by Shearer et al. [6], such a plan must be balanced against signs and symptoms (such as apnea) and indeed, early removal may be necessary. In these unusual cases, the histologic diagnosis agy be incorrect unless electron microscopy is utilized. Fizaliy, this report of a disease-free 7-year follow-up gives some hope that all cases of fibrous histiocytomas may not behave in an uncontrollable, aggressive manner when a complete wide local excision is feasible. 


\section{Re?erences}

1 Batsakis. J.G., Tumors of the Head and Neck. Cinisal and Pathological Considerations, Williams and Willikins, Baltimore, 1974.

2 Blizer, A., Lawson, W. Fahn, F.T., Biller, H.F. and Som, M L., Cinizal-pathological determinants in prognosis of fibrous histiocytomas if the head and neck, Laryngoscope, 91 (1981) 2053.

3 Del-Ray, E. and De-La-Torre, F.E., Fihrous histiccyroma of the nasal cavity, Larymgoscope, 90 (1980) 1686.

4 Kauffman, S.L. and Stout, A.P., Histiocytic tumors (fibrous xanthome and histiocytoma) in children, Cancer: 14 (3) (1961) 469.

s Rice, D.H., Batsakis, J.G., Headington, J.T. and Boles, R., Fibrous histiocytomas of the nose and paranasal sinuses, Arch. Otolaryngol, 100 (1974) 398.

6 Shearer, W.T., Schremir, R.L., Ward, S.P., Masilail, R.E., Strominger, D.B., McAlister, W.H., Kissanc, J. and Ogura, J.H., Benign nasal tumor appearing as neonatal respiratory distress, Ans. J. Dis. Child, 126 (1973) 238.

7 Townsend, G.L., Neel, H.B., Weiland, L.H., Devine, K.D. and McBeau, J.R., Fibrous histiocytoma of the paranasal sinuses, Arch. Otolaryngol., 98 (1973) 51. 